\title{
LA ALFABETIZACIÓN INICIAL CON NIÑOS Y NIÑAS DE 5 A 6 AÑOS. ASPECTOS CLAVE PARA LA FORMACIÓN INICIAL DOCENTE
}

\section{INITIAL LITERACY WITH 5 AND 6 YEAR-OLD CHILDREN. KEY ASPECTS TO INITIAL TEACHING TRAINING}

\author{
Alícia Santolària Òrrios \\ alicia.santolaria@uv.es \\ Facultat de Magisteri. Universitat de València - Grupo GIEL (España)
}

Recibido: 03/03/2021

Aceptado: 31/10/2021

\section{Resumen:}

Se analiza una sesión de aula con niños donde se promueven aprendizajes de aspectos comunicativos de la escritura. Se entiende la alfabetización inicial como un proceso complejo que trasciende la técnica gráfica y se propone estudiar la producción textual como un producto sofisticado. Se diseña una secuencia didáctica implementada en aula, con lo que se obtienen grabaciones de audio transcritas posteriormente. Los resultados muestran, a partir del análisis de categorías emergentes, que los niños acceden a los saberes lingüísticos gracias a que la actividad alfabetizadora se desarrolla en el sociocontructivismo. Finalmente, se exponen los hallazgos y se discuten a la luz de otras investigaciones. Asimismo, se consideran las implicaciones de la investigación para la didáctica de la lengua escrita en los primeros cursos escolares.

Palabras clave: alfabetización inicial; educación infantil; formación docente.

\begin{abstract}
:
A classroom session with children in which learning of communicative aspects of writing is promoted is analysed. Emergent Literacy is understood as a complex process that transcends the graphic technique and aims to study textual production as a sophisticated product. A didactic sequence is implemented in the classroom, with which audio recordings are obtained and are later transcribed. The results show, taking the analysis of emerging categories as a reference, that children access the linguistic knowledge due to the fact that the literacy activity takes place in the socioconstructivism. To conclude, the main findings are presented and discussed in the light of different connected research. In addition, the implications of this research for the didactics of the written language in the first school years are considered.
\end{abstract}

Keywords: emergent literacy; early childhood; teacher training. 


\section{Introducción}

El conocimiento generado sobre alfabetización inicial posterior a 1960 se ha caracterizado por mostrar posturas divergentes, pero desde todas ellas se considera que enseñar a escribir constituye un proceso complejo que va más allá de la mera técnica gráfica y se propone estudiar la producción de un texto como un producto sofisticado. Desde un punto de vista sociocultural, se propone explorar el proceso de construcción de los conocimientos lingüísticos en contexto, situando las prácticas alfabetizadoras en un marco social y cultural donde la comunicación sea el eje interpersonal que articule la enseñanza de la lengua escrita. De hecho, el currículum de Educación Infantil actual recoge los postulados vigotskianos y plantea el acercamiento a la lengua escrita en términos instrumentales donde la promoción de las habilidades comunicativas esté al servicio del uso. El currículo, por tanto, pone el foco en la perspectiva funcional y no solo en cuestiones relacionadas con el sistema de escritura (correspondencia grafía-fonema, identificación de letras o sílabas, etc.). Además, también se pretende conseguir que los niños interpreten los textos en situaciones comunicativas relevantes y próximas para ellos, después de realizar una preparación colectiva previa y con la guía y ayuda del docente (Real Decreto 1630/2006 del marco estatal y Decret 38/2008 de la Comunitat Valenciana).

Dicho esto, desde una perspectiva didáctica, la cuestión estriba en cómo extender en las aulas de los primeros cursos la concepción sociocultural en relación con la lengua escrita, ya que existen prácticas escolares que no han superado el paradigma mecanicista y se resisten a plantear la aproximación a la lengua escrita dentro de un enfoque sociocultural (Sánchez y Santolària Òrrios, 2020; Zamero, 2010). La tensión entre la aproximación a la lengua escrita en las primeras edades escolares desde enfoques formales o enfoques constructivistas sigue latente, aunque la evidencia científica arroja datos sobre la obtención de mejores resultados y prácticas de calidad con el enfoque socioconstructivista (Gil, 2016; Gutiérrez y Sánchez, 2020; Pérez Peitx y Fons Esteve, 2019; Zuccalá y Sandbank, 2018). Este trabajo adopta la perspectiva socioconstructivista de la enseñanza inicial de la lengua escrita proponiendo, desde un punto de vista cualitativo, la descripción y análisis de una sesión de un aula con niños de cinco años donde se despliegan conocimientos textuales sin atender simultáneamente a cuestiones relacionadas con el sistema de escritura, con el propósito de generar conocimiento didáctico que redunde en la formación inicial de maestros, aproximando los contenidos curriculares aludidos con prácticas de calidad dentro del enfoque socioconstructivista.

Consideramos que ahondar en este punto es notable por tres motivos. En primer lugar, se ha de señalar que la investigación en la etapa de Educación Infantil en los últimos 20 años es abundante y trata temas centrados en cuestiones como el análisis de leyes educativas, tipos de centros o familias (Páramo Iglesias, 2016) o la relación entre las familias y uso de las TIC (Campos Bandrés y Marco Sisamón, 2021). Contrariamente, el proceso de alfabetización iniciado en la escuela y la investigación sobre la lengua escrita en los primeros cursos no ocupa un lugar destacado en los estudios generales sobre dicha etapa educativa, a pesar de la relevancia del desarrollo lingüístico para una Educación Infantil (El en adelante) de calidad.

En segundo lugar, a pesar de las diferentes investigaciones sobre alfabetización inicial en la última década, hay que destacar que son pocos los estudios centrados en aula que muestren y analicen con detalle el quehacer didáctico (Sánchez Rodríguez y Santolària Òrrios, 2020). La mayoría de las investigaciones se centran en otras cuestiones como la revisión bibliográfica (Santana et al., 2017); la intervención experimental (Albuquerque y Alves Martins, 2016) o la observación docente (Tolchinsky, Ribera y García-Parejo, 2012).

Por último, dentro de los estudios sobre alfabetización inicial (Al en adelante), se despliega una corriente que explica que se puede acceder a los conocimientos textuales al mismo tiempo, y no después, de resolver el acceso al sistema de escritura alfabético (Ribera, 2008; Ríos García, 
2000). En este trabajo se asume esta perspectiva y se centra en otros logros como el desarrollo de la competencia en comunicación escrita y oral, la familiaridad con los géneros discursivos, la organización de la participación en prácticas letradas compartidas o la calidad de la mediación docente en tareas de lectura y escritura, en línea con los estudios de Fernández (2015); Gutiérrez y Sánchez (2020); Llamazares y Alonso-Cortés (2016); Masgrau y Pons (2019); Pérez Peitx y Fons Esteve, 2019); Santolària Òrrios $(2018,2019)$ y Santos (2018).

El objetivo de este trabajo radica en analizar la enseñanza precoz de los textos. Se quiere saber cómo se enseña a escribir un género discursivo nuevo sin que los niños tracen letras o grafiquen y cómo son las ayudas pedagógicas que ofrece la docente para promover este aprendizaje. Para ello se cuenta con datos orales transcritos que se analizan a partir de categorías temáticas. El análisis de estas categorías, como se verá a lo largo del artículo, demuestra que la práctica en el aula analizada va más allá de una concepción de la escritura como sistema de transcripción y, por tanto, su aprendizaje no se concibe solamente como la adquisición de una técnica, sino que se concibe como un sistema de representación y, por tanto, su aprendizaje se convierte en la apropiación de un nuevo objeto de conocimiento (Ferreiro, 1985). Las consecuencias didácticas de este posicionamiento permiten programar situaciones de escritura con sentido desde los primeros cursos, cuando los niños y las niñas aun no dominan las convenciones del nivel del sistema alfabético.

\section{Diseño de la investigación}

El enfoque metodológico desarrollado en el estudio es cualitativo ya que se analiza un fenómeno que acontece en un contexto determinado, entendiendo que se trata del estudio de fenómenos y experiencias situadas en «un aquí y ahora irrepetibles y de todas ellas se desprenden tensiones porque no existe una única manera de actuar» (Pérez Peitx y Fons Esteve, 2019, p. 170). El planteamiento es coherente con la tradición de investigación en aula en didáctica de la lengua (DL) ya que el estudio cualitativo ayuda a entender los fenómenos acontecidos con profundidad y ofrece información profunda sobre los procesos que permiten generar conocimiento (Camps, 2001; 2006; Castedo, 2007; Gil, 2016; Palou Sangrà y Fons Esteve, 2013; Santolària Òrrios y Ribera, 2017). Se siguió el siguiente procedimiento en la investigación:

1. Selección y desarrollo de contenidos curriculares relacionados con la escritura de textos en edades tempranas.

2. Diseño de una secuencia didáctica (SD) para desplegar la enseñanza-aprendizaje de dichos saberes.

3. Implementación de la SD en aulas de tercero de EI.

4. Investigación de logros y dificultades tras la implementación.

Dada la extensión del artículo, se decide optar por el análisis minucioso de una parte del desarrollo de una sesión de clase, concretamente del primer taller de aprendizaje (véase Anexo), en el que, antes de acometer propiamente la escritura del texto, se plantean en el aula cuestiones poco exploradas en edades tempranas como son los contenidos pragmáticos que permiten vincular el texto en su contexto, aspecto clave en la comunicación. El análisis micro permite, tras la organización de los datos, describir la experiencia, comprender la toma de decisiones e interpretar la intervención para generar conocimiento didáctico.

\subsection{Instrumentos}

Los instrumentos utilizados son diversos: los materiales generados tras el diseño e implementación de la SD, las anotaciones de la investigadora y las grabaciones en audio. Los datos totales registrados suponen 150 minutos de audio. Se seleccionó un registro de 30 
minutos, que fue posteriormente transcrito, donde se promueve la enseñanza y aprendizaje de un texto nuevo para los niños (una ficha técnica). Se trata del primer taller de aprendizaje de la SD (véase Anexo 1) cuyo objetivo es significativo en el marco de la Al: el reconocimiento de las características comunicativas del género, ya que la situación de producción textual comienza mucho antes de la alfabetización mecánica (Camps, 1994; Ferreiro, 1999, 2001; Ribera, 2013; Sánchez Rodríguez, 2014; Teberosky y Tolschinsky, 1995).

Los datos han sido captados en dos niveles de análisis: en el primer nivel, se estudia la sesión seleccionada y se distinguen las categorías Número de turnos y Andamiajes; en el segundo, se analizan los diálogos y se distingue la categoría Enfoque en la tarea. Las categorías se desplegarán posteriormente en subcategorías (Tabla 1). La información obtenida revela cómo se lleva a cabo la enseñanza de los contenidos y cómo son aprehendidos por el grupo clase.

Tabla 1

Niveles de análisis

\begin{tabular}{|l|l|}
\hline Nivel 1: Análisis sesión & Nivel 2: Análisis diálogos en aulas de primeros cursos \\
\hline Número de turnos & Enfoque tareas \\
Andamiajes & Perspectiva significativa \\
Dictado al docente & Atención al proceso \\
& Relación lectura y escritura \\
\hline
\end{tabular}

Fuente: elaboración propia

La primera categoría del primer nivel se refiere a la cantidad de turnos de palabra usados por el grupo clase (por la maestra y por el grupo). Por Andamiajes se entiende el conjunto de ayudas pedagógicas que la maestra ofrece para hacer avanzar el conocimiento (Bruner, 1989). En el segundo nivel se analizan los intercambios lingüísticos producidos en el aula a través de los cuales se observa cómo se enfocan las tareas y cómo se desarrolla el ambiente de escritura.

Es significativa la fecha de recogida de datos (final de curso) y el carácter de la SD, enmarcada en un enfoque sociocultural orientado a la promoción de actividades comunicativas, de manera que para que los niños tomen conciencia de la necesidad de realizar textos se piensa en crear una exposición escolar con todos los escritos.

\subsection{Participantes}

Intervienen 25 niños y niñas y una maestra de una escuela pública valenciana. Se trata de un aula multicultural donde las edades oscilan entre los cinco y seis años. Los niños se escolarizan exclusivamente en catalán hasta segundo de Educación Primaria, según los criterios pedagógicos del centro, que promueve la enseñanza de la lengua minorizada. Los diálogos originales, por tanto, son en catalán. Se han traducido al español para facilitar su comprensión.

\section{Resultados}

Se presenta el análisis organizado en dos niveles: el primero ahonda en el desarrollo de la sesión seleccionada y el segundo en el carácter de los diálogos. Se incluyen citas textuales marcando la intervención de la maestra con una M. Las intervenciones de los niños van precedidas con otras letras, que representan sus nombres. Por último, se indica el número del turno.

\subsection{Análisis de la sesión}

En la Tabla 2 se observa el desarrollo de la sesión con las acciones realizadas y en la figura posterior la distribución de los turnos por fase. 
Tabla 2

Descripción de la sesión seleccionada

\begin{tabular}{|c|c|c|c|}
\hline Fase & Acciones & $\begin{array}{l}\text { Turno } \\
\text { palabra }\end{array}$ & Desarrollo \\
\hline \multirow[t]{5}{*}{1} & Contextualización tarea & $1-14$ & Repaso sesión anterior \\
\hline & & 15 & $\begin{array}{l}\text { La maestra anuncia la tarea de escritura del proyecto: } \\
\text { elaborar la ficha técnica sobre un cuento para una } \\
\text { exposición escolar. } \\
\text { Utiliza tres textos (modelo de fichas técnicas). } \\
\text { Los cuelga en la pizarra e invita a los niños a formular } \\
\text { hipótesis sobre el contenido temático de los escritos. }\end{array}$ \\
\hline & $\begin{array}{l}\text { Introducción textos } \\
\text { modelo }\end{array}$ & $16-26$ & $\begin{array}{l}\text { Hipótesis, lectura por parte de la maestra y diálogo } \\
\text { sobre el texto } 1\end{array}$ \\
\hline & & $27-35$ & $\begin{array}{l}\text { Hipótesis, lectura por parte de la maestra y diálogo } \\
\text { sobre el texto } 2\end{array}$ \\
\hline & & $36-47$ & $\begin{array}{l}\text { Hipótesis, lectura por parte de la maestra y diálogo } \\
\text { sobre el texto } 3\end{array}$ \\
\hline \multirow[t]{7}{*}{2} & $\begin{array}{l}\text { Reflexión sobre algunos } \\
\text { parámetros de la } \\
\text { comunicación a partir de } \\
\text { textos modelo } \\
\text { (destinatario y función) }\end{array}$ & 48 & $\begin{array}{l}\text { La maestra plantea una nueva tarea sobre los textos } \\
\text { leídos. Anotará lo que los niños dirán en la pizarra } \\
\text { (dictado al docente). Acuerdan escribir tres } \\
\text { conclusiones por texto. }\end{array}$ \\
\hline & & $49-50$ & Los niños aceptan la propuesta de la maestra. \\
\hline & $\begin{array}{l}\text { Reflexión oral sobre la } \\
\text { tarea propuesta. Dictado } \\
\text { al docente }\end{array}$ & 51 & $\begin{array}{l}\text { La maestra pregunta para quién, para qué se ha escrito } \\
\text { el texto } 1 .\end{array}$ \\
\hline & & $52-79$ & $\begin{array}{l}\text { Conversación sobre el texto } 1 \text {. Los niños explican por } \\
\text { qué se ha escrito. }\end{array}$ \\
\hline & & $80-90$ & $\begin{array}{l}\text { Conversación sobre el texto } 2 \text {. Los niños explican por } \\
\text { qué se ha escrito. }\end{array}$ \\
\hline & & $99-118$ & $\begin{array}{l}\text { Conversación sobre el texto 3. Los niños explican por } \\
\text { qué se ha escrito. }\end{array}$ \\
\hline & & $121-182$ & $\begin{array}{l}\text { Los niños y la maestra leen y revisan lo que hay escrito } \\
\text { en la pizarra. }\end{array}$ \\
\hline \multirow[t]{3}{*}{3} & $\begin{array}{l}\text { Explicación del género } \\
\text { meta a partir de } \\
\text { reflexiones anteriores }\end{array}$ & 183 & $\begin{array}{l}\text { La maestra explica a los niños que los textos leídos son } \\
\text { fichas técnicas, recuerda para qué sirven y conecta lo } \\
\text { que los niños han aprendido con el texto que tendrán } \\
\text { que escribir entre todos a lo largo de la semana para la } \\
\text { exposición de cuentos en la escuela. }\end{array}$ \\
\hline & & 184 & $\begin{array}{l}\text { Conversación entre maestra y niños sobre las } \\
\text { características del género. }\end{array}$ \\
\hline & $\begin{array}{l}\text { Reconocimiento del } \\
\text { género y planteamiento } \\
\text { de la siguiente tarea }\end{array}$ & $210-217$ & $\begin{array}{l}\text { La maestra explica que han aprendido el género e } \\
\text { insiste en que tendrán que escribir uno igual durante } \\
\text { los próximos días. }\end{array}$ \\
\hline
\end{tabular}

Fuente: elaboración propia 
Figura 1

Fases y turnos de palabras

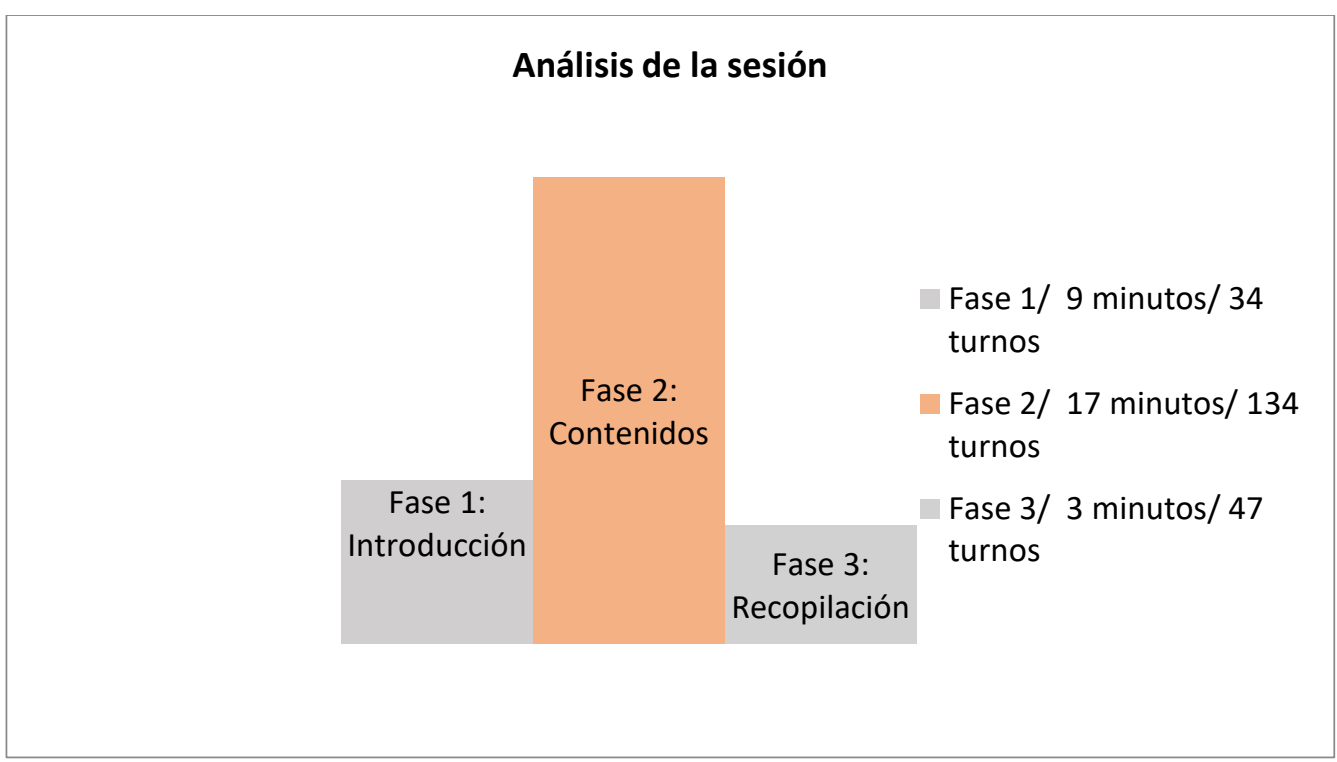

Se puede observar que la sesión elegida se divide en tres fases: en la fase 1 la maestra conecta con los conocimientos previos del grupo, apela a la memoria de los niños y realiza preguntas concretas sobre el cuento leído previamente:

M: ¿el protagonista de la historia de ayer era un chico o una chica? (1). ¿Cómo era? (7)

El contenido de la segunda fase se articula sobre algunos parámetros de la comunicación, especialmente sobre el destinatario. En el desarrollo de dicha fase se emplean diecisiete minutos donde la maestra plantea, a partir de los textos leídos, para qué, por qué y para quién han sido escritos:

$M: ~ ¿ p a r a$ qué pensáis/bien/ para qué pensáis eh que han escrito esta ficha técnica/ este texto/ para qué/ o para quien/ con qué finalidad/ para qué/ han escrito/ para qué pensáis? (51).

La estrategia recurrente usada para lograr la aprehensión de los contenidos demandados es la pregunta explícita. El análisis de la sesión muestra que los niños tienen mayor dificultad para identificar los aspectos pragmáticos que los temáticos. La maestra observa dichas dificultades y realiza primero preguntas abiertas y después reformulaciones, como se observa en el siguiente fragmento:

M: esa es la información que nos da/ ¿Vale? Esa es la información que nos da/ Eso ya lo sabemos pero ¿Para qué/ ¿Para qué nos han contado esta información del perro? ¿Poner la fotografía del perro y preguntar si lo queremos adoptar o apadrinar? ¿Para qué? Úrsula/ piénsatelo/ Lucía (86)

L: para que adoptemos a un perro (87)

La última fase es la más breve (tres minutos). En ella la maestra recuerda el propósito de la tarea:

M: porque así podremos dar información de todo lo que nosotros queramos/de forma muy fácil y clara/ vamos a aprender estos días (217) 


\subsubsection{Número de turnos}

El análisis permite identificar 217 turnos de palabra ( 30 minutos de grabación) de los cuales un $49,3 \%$ corresponden a la maestra y un $50,7 \%$ a los niños. En la fase 2, como se ve en la Figura 1, es donde más turnos confluyen ya que en ella se trabajan los contenidos nuevos para el grupo (la dimensión comunicativa de los textos).

\subsubsection{Andamiajes: los modelos y el dictado al docente}

La maestra apoya el proceso de enseñanza y aprendizaje del género, que en estas primeras edades es muy demandante. Ofrece modelos, como se ve a partir del turno 15 , cuando se desarrolla una tarea donde la docente muestra tres textos expuestos en la pizarra. Se trata de tres fichas técnicas que aparecen en la vida cotidiana de los niños (la contraportada de un libro de la biblioteca del aula, un anuncio de un animal de la protectora y otro sobre un centro escolar). Tras la lectura realizada por la maestra, los niños realizan hipótesis sobre el contenido de los textos:

M: tenemos el Texto 1 voy a poner aquí un numerito para aclararnos/ el uno/ el dos y el tres/ a ver/ antes de yo comentar y leer nada/ así/ a primer golpe de vista/ este texto ¿tú de qué crees que hablará? (15)

G: de dinosaurios (16)

M: de un dinosaurio y ¿qué puede/ ¿Qué puede pasar a los dinosaurios? (17)

El dictado al docente ahonda en el potencial didáctico que supone anteponer la enseñanza de los aspectos textuales a los del sistema de escritura. Se sabe que, en las primeras edades, abordar dichas enseñanzas a la vez podría sobrecargar cognitivamente a los niños. Se trata de una forma de escritura que comporta grandes beneficios y que ha sido estudiada en edades precoces (Aeby y Almgren, 2018; Santos, 2018). Los niños explican oralmente al docente lo que se ha de escribir y este traza las letras. En la Imagen 1 también se pueden observar las ideas que los niños aportan sobre los textos y que la maestra anota en la pizarra. Veamos el siguiente fragmento:

M: con esa información que tenemos ahí/ dirección e información de la guardería/ un autobús... ¿Para qué? (101)

G: para sirve para saber dónde estamos (102)

M: para saber dónde estamos (103)

G: No cabe (104)

M: sí que cabe (105)

X: sí que cabe (106)

$\mathrm{L}$ : donde (107)

M: venga/alguna cosa más? Izan/para qué piensas que esta información/ que hay aquí ¿para qué piensas que la han hecho? Han puesto guardería/ plano/ un autobús/ ¿para qué? (108)

I: es una guardería (109)

M: Granada dice que para saber dónde estamos (110)

$\mathrm{X}$ : para ir a los sitios (111)

M: para ir a los sitios (112)

$X:$ nooo (113)

M: y ahora pregunto yo/ ¿para ir a los sitios? ¿A unos sitios? ¿O un sitio determinado? ¿Tú qué crees? ¿Será para ir dónde? (114)

M: ¿qué lugar será ese? (116)

X: la guardería (117)

M: la guardería (escribe en la pizarra) (118) 
Imagen 1

Ejemplos de textos

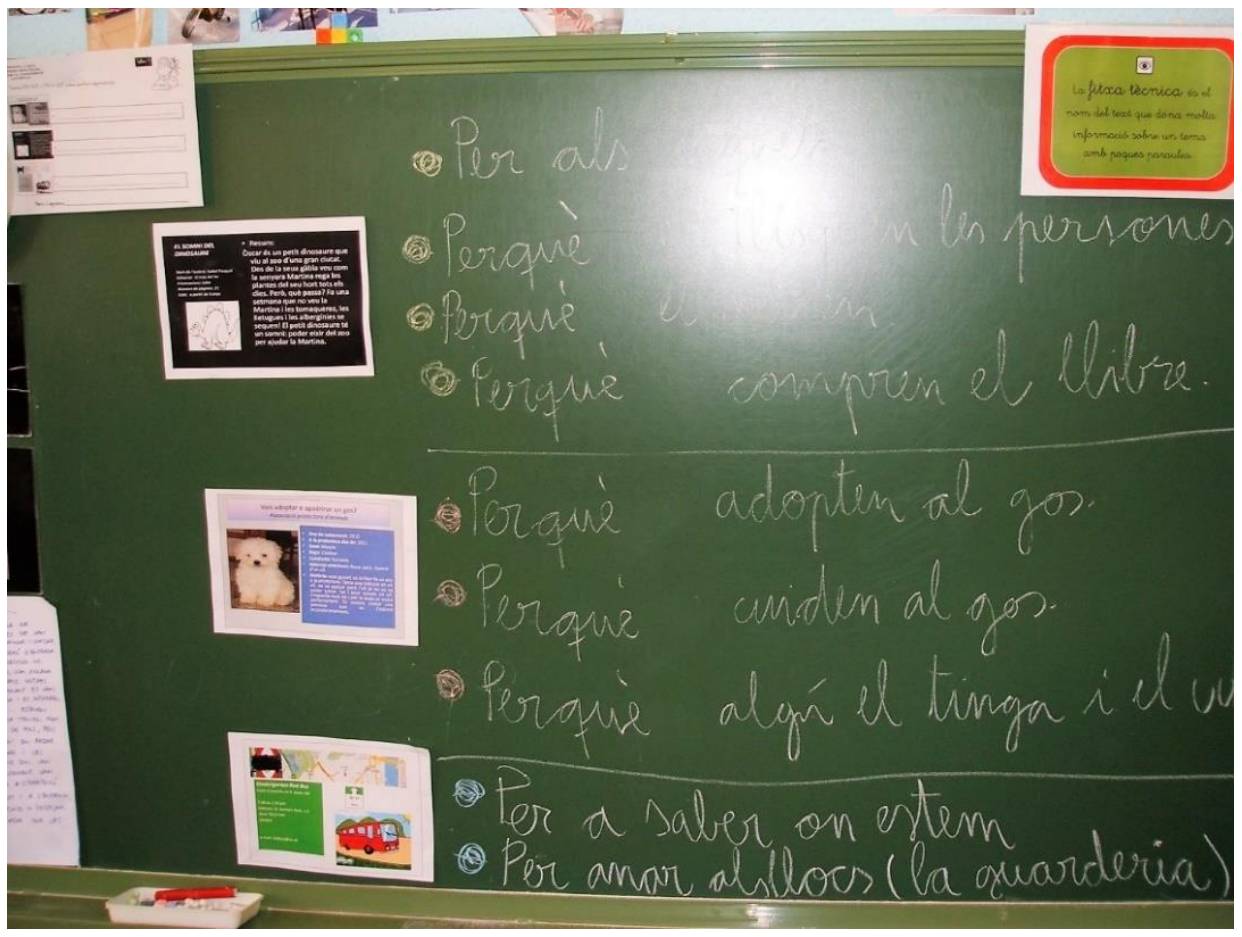

La sesión analizada revela que la actividad alfabetizadora se desarrolla en un escenario sociocultural donde una actividad dialógica organizada permite partir de las hipótesis de los niños sobre el objeto de conocimiento y generar aprendizajes (Rojas-Drummond et al., 2020; Rojas-Drummond y Mercer, 2003; Wells, 2015). La conversación pivota, primeramente, sobre la comprensión de los textos ( $M$ : este texto/ ¿tú de qué crees que hablará? -15) y, en segundo lugar, sobre los parámetros de la comunicación (X: para que compren el libro (T-74) Para que lo lean (T-58). Además, el diálogo genera una reflexión sobre las características del género que van a escribir. Cabe destacar que los niños entienden una de las características fundamentales: debe ser cortito (194), añaden que son 4 palabras (199).

\subsection{Análisis de los diálogos en aulas de primeros cursos}

El análisis de la interacción verbal goza de gran relevancia y trayectoria entre las teorías socioculturales. En este caso, los intercambios lingüísticos producidos en aula se analizan según los trabajos de Alonso-Cortés y Sánchez (2018) quienes actualizan dicha trayectoria y se centran en la elaboración de instrumentos para observar prácticas de aula que consideren de forma integrada la naturaleza de las tareas de producción textual y las diferentes habilidades y procesos lingüísticos en los primeros años.

El diálogo se entiende como una situación comunicativa que contribuye al aprendizaje de los contenidos promovidos. La maestra, en este registro, dirige las intervenciones e invita a participar y a elaborar hipótesis de dos formas: nombra a los niños que levantan la mano y también se dirige a todo el grupo. Solo en una ocasión un niño parte del enunciado de otro. En alguna ocasión responden a coro y generalmente los niños responden a la demanda de la docente. Para captar la atención e implicar a los niños la maestra realiza preguntas y en ocasiones correcciones implícitas: 
M: pero es un resumen de un/ si habla de páginas/ de ilustraciones/ de editoriales/ ¿será un resumen de un? (25)

M: para los cuentos/ Mmmmm... pensad un poquito más/ ¿para qué? (55)

\subsubsection{Enfoque de la tarea}

Las tres subcategorías incluidas en esta dan cuenta del enfoque: se concibe la actividad desde una perspectiva significativa, que atiende al proceso y que relaciona la lectura con la escritura. Además, es importante señalar que la docente crea un clima afectuoso donde anima a los niños a participar. Se da tiempo y da tiempo para que los niños interactúen y razonen con los materiales. De hecho, los resultados muestran que se usan 17 minutos en la fase dos, donde los niños encuentran mayores dificultades.

\subsubsection{Perspectiva significativa y atención al proceso}

La meta de la maestra es que los niños y las niñas aprendan al final del proceso a realizar un texto desde un punto de vista significativo y para ello expresa de forma organizada la tarea, como se ha visto en el análisis del nivel 1 . Antes deben de conocer muchos aspectos textuales como las características del género que han de escribir. Así, la docente explicita el objetivo global de la actividad y a continuación explica de forma implícita que se hallan ante un proceso.

$M$ : vosotros tenéis que saber que el cuento que estamos aprendiendo y que conté ayer/ nosotros después lo tenemos que contar y pasar la información de toda esa historia a los mayores para que conozcan la historia de la niña sabia y lo haremos de una forma pues muy clara/con una ficha técnica/ ¿vale?/por tanto/mirad aquí tenemos tres fichas/ después haremos unas fichitas que hemos preparado/ tenemos el Texto 1 voy a poner aquí un numerito para aclararnos/ el uno/ el dos y el tres/ a ver/ antes de yo comentar y leer nada/ así/ a primer golpe de vista/ este texto tú de qué crees que hablará (15)

Se concibe, por tanto, la tarea como un proceso y no como el resultado de una instrucción directa para elaborar un producto final. Los niños emplean 31 turnos de palabra (de los 217 de toda la sesión) para entender primero y escribir mediante el dictado al docente después para qué sirve el primer texto (turnos entre 48-79), 18 para el segundo (turnos 80-98), y 21 para el tercero (turnos 99-120). En la Figura 2 se puede observar la relación entre los textos y los turnos de palabra empleados. 
Figura 2

Relación textos y turnos de palabras

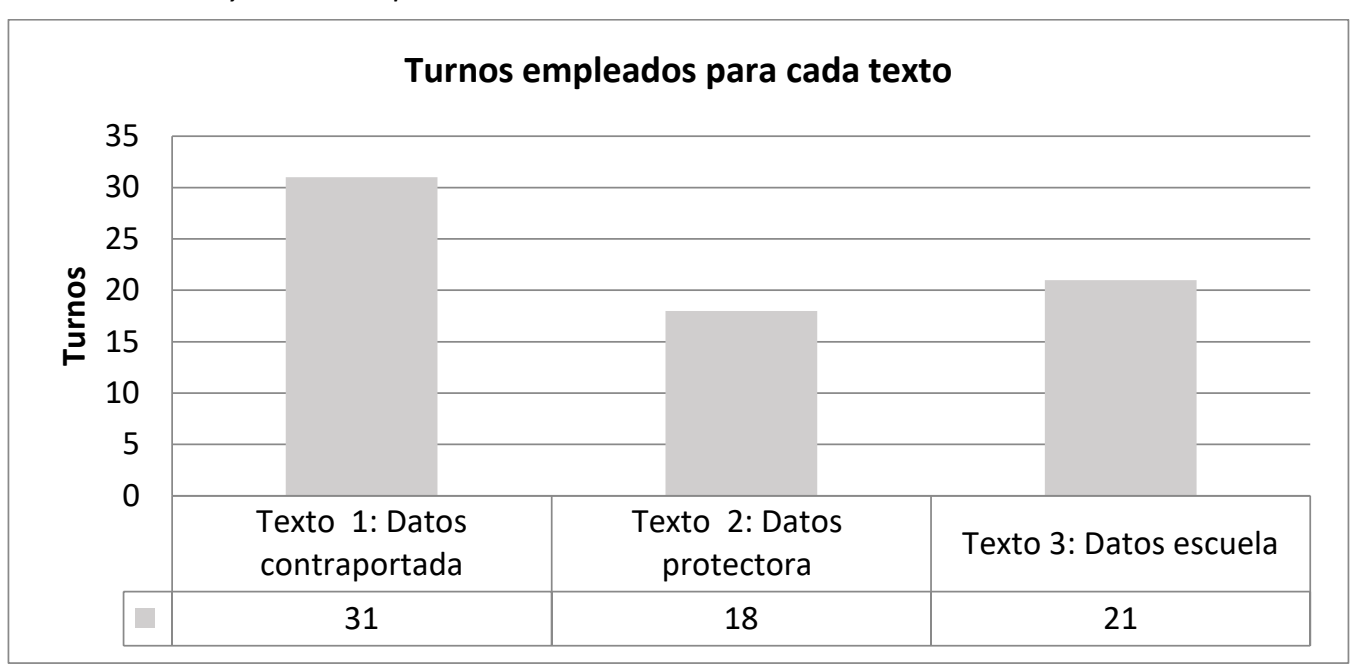

\subsubsection{Relación lectura y escritura}

En la primera alfabetización los niños trabajan lectura y escritura de manera complementaria, ya que se entiende que estas tareas se han de relacionar con los usos comunicativos y sociales. Esta práctica efectiva se va abandonando en los cursos superiores porque a menudo se parcelan desvinculando las operaciones lectoras de las habilidades escriturales (Guasch y Fontich, 2012). En este estudio, la maestra, además de interrelacionar las dos competencias, pone el acento en la comprensión activa y funcional de los escritos y permite, antes de descodificar los textos, que los niños formulen hipótesis, esto es, posibilita una aproximación a la lengua escrita a partir de la comprensión y de las inferencias, no de la descodificación del sistema de escritura. Este hecho permite a los niños ser receptores y emisores de los textos al mismo tiempo, como se puede ver en el fragmento:

M: de un libro o un cuento/ lo que pasa es que nos han dado una información importante de forma muy cortita sobre ese cuento así si yo leo el resumen y me gusta puedo decidir si me lo compro/ es un resumen de un cuento/ ya lo tenemos/ ese ya sabemos de qué habla/ de un cuento/vale/ después tenemos otro texto/ el número 2 y dice/voy a leerlo que esta letra es más pequeña/ bueno os imagináis ¿de que irá? ¿De qué ira? (27)

Niños (a coro): de un perro (28)

M: de un perro/ tenemos la fotografía/ esta fotografía ya nos da información pero además la acompañan una serie de textos que a ver qué dicen (la maestra lee) ¿Quieres adoptar o apadrinar un perro? Asociación protectora de animales y dice año de nacimiento 2010/ ¿del nacimiento de quién? (29)

Niños (a coro): del perro (30)

\section{Discusión y conclusiones}

Los resultados se han agrupado en torno a dos niveles de análisis. Dentro del primero, la categoría Número de turnos muestra que la maestra se apropia de casi la mitad de los intercambios lingüísticos. Este resultado entronca con los hallados en otras investigaciones socioconstructivistas en las que se observan participaciones equilibradas entre niños y docentes (Ribera, 2008; Ríos García, 1999). Así, la investigación psicoeducativa ha mostrado evidencias de cómo el aprendizaje cooperativo mejora el desarrollo y el rendimiento académico (Lago y Naranjo, 2016). El papel del docente no es de transmisor del saber y del conocimiento, sino que 
orienta el discurso oral y provoca interrogantes, gestiona la conversación, cohesiona y dota de sentido las diferentes aportaciones de los niños para que enriquezcan los aprendizajes individuales y colectivos (Sanz Datzira y Monterde i Farnés, 2011).

Entendemos con Camps (2017b) que la interacción caracteriza el proceso de aprendizaje y, en este sentido, en este artículo, se pone el foco en las ayudas o andamiajes que requieren de las habilidades orales como el dictado al docente, que posibilita la producción textual sin necesidad de codificar, descodificar o graficar. Autoras como Canut (2009) señalan que el grafismo tal vez represente un freno en un principio, que puede ser resuelto cuando el adulto se convierte, de alguna manera, en el bolígrafo del aprendiente. Los resultados hallados conectan con otros en los que se valoriza esta actividad lingüística que permite trabajar la entrada al escrito y ahonda en el potencial didáctico que supone anteponer la enseñanza de los aspectos textuales a los del sistema de escritura (Aeby y Almgren, 2018; Ribera, 2008; Santolària Òrrios, 2019; Santos, 2018), aspecto de implicaciones didácticas importantes ya que la evidencia científica muestra que los niños se fijan antes en las características más generales de los materiales impresos de su alrededor que en las marcas gráficas concretas como las letras (Gil, 2016).

El segundo nivel se centra en los diálogos en aulas de primeros cursos, eje de la DL en tanto en cuanto estudia la conversación como generadora de procesos de aprendizaje. Del análisis de la categoría Enfoque de la tarea se constatan dos aspectos relevantes: la docente plantea la escritura en grupo y de forma conversacional. Este procedimiento entronca con las teorías socioculturales que, en oposición a las prácticas más habituales hasta hace algunos decenios, establecen la idea de que escribir no es una actividad solitaria, sino que los textos se relacionan con otros de forma contextualizada (Ribas Seix, Rodríguez-Gonzalo y Durán, 2020). Hay otros dos puntos relacionados con este: la perspectiva significativa y la atención al proceso. Plantear la necesidad de escribir se erige como el asunto nuclear de la didáctica de la Al, centrada en dotar de sentido las prácticas de lectura y escritura, de manera que el enfoque funcional cobra mayor relevancia que la preferencia por cualquier tipo de método. Relacionado con este punto, es trascendental la representación del destinatario en la tarea. Los resultados de este estudio muestran que los niños tienen dificultades para transitar entre las posiciones de emisor y receptor, que se superan gracias a los andamiajes ofrecidos. Dichos andamiajes sitúan la actividad de escritura en un contexto donde hay un emisor, un destinatario y un propósito que otorga sentido a la tarea.

Otra cuestión destacable es la atención al proceso. En este estudio hemos podido observar que la docente concibe la escritura no solo como un producto final, sino también como un proceso, un acto en curso que se planifica oralmente. La atención a los procesos de escritura es un tema recurrente en la DL desde los años 80 del pasado siglo (Camps, 2017c). Por otro lado, en la sesión analizada, la relación entre lectura y escritura es constante. El foco se sitúa en la comprensión para obtener una visión esencial del contenido y no en la codificación y descodificación (Clemente y Rodríguez, 2014; Palou Sangrà y Fons Esteve, 2016).

El análisis revela que la docente media entre el objeto de conocimiento y los niños, de la misma manera que docentes y adultos median entre la lectura de cuentos y los niños (Trigo-lbáñez, Rivera-Jurado y Sánchez-Rodríguez, 2020). Así, la lectura mediada por adultos en los primeros años resulta fundamental para el desarrollo lector en la escuela (Santana et al., 2017), de la misma manera que el diálogo y el dictado al docente, como estrategias didácticas, constituyen herramientas de aproximación a la lengua escrita idóneas en edades tempranas. Esto se realiza en un ambiente en el que la maestra estimula la comunicación, donde se resalta el valor educativo de la conversación y la interacción (Fontich y Fabregat, 2021; García-Carrión y Villardón-Gallego, 2017; Masgrau y Pons, 2019; Sanz Datzira y Monterde i Farnés, 2011). 
Asimismo, el registro de sesiones como instrumento de recogida de datos resulta válido para responder a los objetivos planteados en este estudio: describir y analizar una sesión de aula donde se promueven contenidos curriculares sobre la producción escrita en edades tempranas, priorizando los aspectos comunicativos en detrimento de los relacionados con la actividad de graficar. El debate sobre si conviene o no esperar a abordar la composición textual hasta que los niños estén ya en la etapa alfabética y posean conocimientos sólidos sobre el sistema de escritura ha sido superado por los avances científicos, si bien se encuentran ciertas resistencias en algunas aulas (Clemente y Rodríguez, 2014; Gil, 2016; Sánchez y Santolària Òrrios, 2020).

El análisis de sesiones a través de registros permite formular cuestiones como las planteadas por Castedo (2007): qué se pretende enseñar, qué situaciones se presentan para enseñar esos contenidos, cómo se organiza el grupo, etc. Se entiende así la DL no solo como una disciplina de intervención, sino también como una disciplina de análisis de la práctica docente (Camps, 2012). El registro permite detallar cómo se desarrolla una sesión y ofrece reflexión sobre la praxis. Justamente este es uno de los escollos que pretende superar dicha disciplina: la falta de investigaciones y materiales que ofrezcan sesiones de referencia para analizar prácticas determinadas que expliciten modelos didácticos de calidad, ya que se sabe poco de lo acontecido en las aulas (Viera y Barrio, 2012). Con todo, en los primeros cursos escolares, contamos con estudios enmarcados en propuestas pedagógicas sociocontructivistas que, en relación con la $\mathrm{Al}$, permiten observar quehaceres y proponer discusiones sobre su sentido pedagógico. En este sentido destacamos, entre otros, los estudios de Altava Rubio, Gimeno Agost, Pérez Serrano, Ríos García y Gallardo Fernández (2006), Barrio, Calleja Largo y Garrán Antolínez (2006); Masgrau y Pons (2019); Sánchez Rodríguez y González Aragón (2016); Santolària Òrrios y Sánchez (2021) y Sanz Datzira y Monterde i Farnés (2011).

En definitiva, la praxis del aula va más allá de la concepción de la escritura como resultado de la conversión de códigos y transmite que las cuestiones metodológicas están relacionadas con el marco epistémico de la escritura. Por tanto, este trabajo se suma a la corriente que defiende la alfabetización temprana que antepone los contenidos discursivos ante los mecánicos, contemplando a los niños como agentes activos de aprendizaje, cuyo pensamiento no se debe subestimar (Ferreiro, 2018). La bibliografía coincide en que la Al es el período más importante para el desarrollo de la alfabetización. Con este trabajo se quiere reflexionar sobre esto y generar conocimiento para una formación docente en alfabetización inicial.

\section{Referencias}

Aeby, S. y Almgren, M. (2018). "Le loup entre dans la cage...». Le point sur les apports de la dictée à l'adulte à la production de texte avec de jeunes élèves. Forumlecturech, 1, 1-13.

Albuquerque, A. y Alves Martins, M. (2016). Fomento de habilidades de lecto-escritura en la primera infancia: estudio de seguimiento desde la educación infantil al primer curso de educación primaria. Infancia y Aprendizaje, 39(3), 592-625.

Alonso-Cortés, M. D. y Sánchez, S. (2018). Una guía para observar las prácticas de aprendizaje y enseñanza de la composición escrita en Educación Primaria. Ponencia presentada en el 360 Congreso Internacional de AESLA. Cádiz.

Altava Rubio, V., Gimeno Agost, F., Pérez Serrano, I., Ríos García, I. y Gallardo Fernández, I. M. (2006). El análisis de situaciones de aula como instrumento para la formación del profesorado. En A. Camps (Coord.). Diálogo e investigación en las aulas. Investigaciones en didáctica de la lengua (pp. 263-285). Graó. 
Barrio, J. L., Calleja Largo, I. y Garrán Antolínez, M. L. (2006). La lengua como vehículo para enseñar lengua. En A. Camps (Coord.). Diálogo e investigación en las aulas. Investigaciones en didáctica de la lengua (pp. 55-76). Graó.

Bruner, J. (1989). Acción, pensamiento y lenguaje. Alianza Psicología.

Camps, A. (1994). L'ensenyament de la composició escrita. Barcanova.

Camps, A. (Coord.) (2001). El aula como espacio de investigación y reflexión. Investigaciones en didáctica de la lengua. Graó.

Camps, A. (Coord.) (2006). Diálogo e investigación en las aulas. Investigaciones en didáctica de la lengua. Graó.

Camps, A. (2012). La investigación en didáctica de la lengua en la encrucijada de muchos caminos. Revista Iberoamericana de Educación, 59, 23-41.

Camps, A. (2017a). Escribir es participar en una sociedad alfabetizada. Bases para la enseñanza de la escritura en la educación obligatoria (I). Textos. Didáctica de la Lengua y la Literatura, 75, 32-39.

Camps, A. (2017b). Escribir y aprender a escribir, dos actividades que se entrecruzan. Bases para la enseñanza de la escritura en la educación obligatoria (II). Textos. Didáctica de la Lengua y la Literatura, 75, 40-46.

Camps, A. (2017c). 25 anys de recerca en didàctica de la lengua. Bellaterra Journal of Teaching \& Learning Language \& Literature, 10(1), 9-19. https://doi.org/10.5565/rev/jt|3.721

Campos Bandrés, I. O. y Marco Sisamón, A. (2021). Exploración de la relación entre la alfabetización familiar, las TIC y la competencia lectoescritora. Tejuelo, 33, 161-184. https://doi.org/10.17398/10.17398/1988-8430.33.161

Canut, E. (2009). Apprendre à parler pour ensuite apprendre à lire et à écrire - Pour une théorisation de la question linguistique du passage de l'oral vers l'écrit. https://hal.archives-ouvertes.fr/hal-00524227/document. (Fecha de acceso: 25.10.2021).

Castedo, M. (2007). Notas sobre la didáctica de la lectura y la escritura en la formación continua de docentes. Lectura y Vida. Revista latinoamericana de lectura, 28(2), 6-19.

Clemente, M. y Rodríguez, I. (2014). Enseñanza inicial de la lengua escrita. De la teoría a la práctica. Aula, 20, 105-121.

DECRET 38/2008, de 28 de març, del Consell, pel qual s'establix el currículum del segon cicle de l'Educació Infantil a la Comunitat Valenciana. http://www.dogv.gva.es/datos/2008/04/03/pdf/2008_3838.pdf

Fernández Martínez, P. (2015). Secuencias didácticas para escribir textos expositivos en Educación Infantil [Tesis doctoral. Universidad Complutense de Madrid]. https://eprints.ucm.es/40347/1/T38068.pdf

Ferreiro, E. (1985). A representação da linguagem e o processo de alfabetização. Cadernos de Pesquisa, 52, 7-17.

Ferreiro, E. (1999). Cultura escrita y educación. Conversaciones de Emilia Ferreiro. Fondo de Cultura Económica.

Ferreiro, E. (2001). Pasado y presente de los verbos leer y escribir. Fondo de Cultura Económica.

Ferreiro, E. (2018). Acerca de las dificultades para aceptar que los niños piensan sobre lo escrito. Bellaterra Journal of Teaching \& Learning Language \& Literature, 11(2), 13-34. https://doi.org/10.5565/rev/jtl3.769 
Fontich, X. y Fabregat, S. (2021). Escuela e interacción social: Hablar para pensar, para aprender, para ser. En F. Pesántez-Avilés, L. Álvarez-Rodas y Á. Torres (Comps.). COVIDA-20 Una coalición educativa para enfrentar la pandemia (pp. 55-66). Pearson Educación.

García-Carrión, R. y Villardón-Gallego, L. (2017). La interacción y el diálogo para una educación infantil de calidad. Aula de Infantil, 89, 31-34.

Gil, R. M. (2016). Interpretar i millorar les escriptures infantils: dificultats i reptes. Llengua, Societat i Comunicació: Revista de Sociolingüística de la Universitat de Barcelona, 14, 3037. https://doi.org/10.1344/LSC-2016.14.5

Guasch, O. y Fontich, X. (2012). La lectura a les activitats d'escriptura. Articles de Didàctica de la Llengua i la Literatura, 56, 5-7.

Gutiérrez, M. I. y Sánchez, S. (2020). Escritura autónoma y nivel de uso del sistema de escritura en el último curso de Educación Infantil: Estudio de un caso. Lenguaje y textos, 51, 107119. https://doi.org/10.4995/lyt.2020.12171

Lago, J.R. y Naranjo, M. (2016). Aprendizaje cooperativo en el aula y en el centro. Aula, 255, 5357.

Llamazares, M. T. y Alonso-Cortés, M. D. (2016). Lectura compartida y estrategias de comprensión lectora en Educación Infantil. Revista Iberoamericana de Educación, 71, 151172.

Masgrau, M. y Pons, M. (2019). Narrativas en los inicios de la escritura. La importancia del diálogo y la reflexión en la alfabetización. Textos. Didáctica de la Lengua y de la Literatura, 85, 53-61.

Palou Sangrà, J. y Fons Esteve, M. (2013). Investigar en un escenario denominado aula. Cultura y Educación, 25(4), 489-494.

Palou Sangrà, J. y Fons Esteve, M. (2016). La competencia lectora. En J. Palou Sangrà y M. Fons Esteve (Coords.). Didáctica de la lengua y la literatura (pp. 113-127). Síntesis.

Páramo Iglesias, M. B. (2016). Qué investigar tras veinte años de investigación en Educación Infantil en el ámbito hispánico. Revista Latinoamericana de Educación Infantil, 5(2), 158165.

Pérez Peitx, M. y Fons Esteve, M. (2019). Caracterización de las pautas de acción en la didáctica de la alfabetización inicial. Tejuelo, 30, 151-174. https://doi.org/10.17398/19888430.30 .151

REAL DECRETO 1630/2006, de 29 de diciembre, por el que se establecen las enseñanzas mínimas del segundo ciclo de Educación infantil. (BOE 4-1-2007).

Ribas Seix, T., Rodríguez-Gonzalo, C. y Durán, C. (2020). El aprendizaje de la escritura de textos de opinión en alumnos de Primaria: análisis de la actividad metalingüística, de los conceptos sobre la escritura y de los productos finales. Indagatio Didactica, 12(2), 33-54. https://doi.org/10.34624/id.v12i2.17436

Ribera, P. (2008). El repte d'ensenyar a escriure. Perifèric edicions.

Ribera, P. (2013). Aprender el sistema de escritura alfabético a partir del uso de textos en los inicios de la lectura y escritura. En L. Álvares Pereira (Coord.), Reflexão sobre a escrita. $O$ ensino de diferentes géneros de textos (pp. 95-106). Universidade de Aveiro.

Ríos García, I. (1999). Les intervencions de la mestra en el procés de planificació del text escrit. [Tesis doctoral. Universitat Jaume I]. https://www.tdx.cat/handle/10803/10364 
Ríos García, I. (2000). Planificar vol dir també escriure. Articles de Didàctica de la Llengua i la Literatura, 21, 99-108.

Rojas-Drummond, S., Barrera, M. J., Hernández, I. Alarcón, M. Hernández, J. y Márquez, A. M. (2020). Exploring the 'black box': What happens in a dialogic classroom. Australian Journal of Language and Literacy, 43, 47-67.

Rojas-Drummond, S. y Mercer, N. (2003). Scaffolding the development of effective collaboration and learning. International Journal of Educational Research, 39, 99-111.

Sánchez Rodríguez, S. (2014). La alfabetización inicial en los últimos 25 años: de los métodos de lectura a las prácticas docentes para enseñar a leer y escribir. En M. Romero; R. Jiménez (Coords.). Hacia una educación lingüística y literaria: Homenaje a los 25 años del Departamento de Didáctica de la Lengua y la Literatura (1987-2012) (pp. 177-198). UCA.

Sánchez Rodríguez, S. y González Aragón, C. (2016). La asamblea de clase en Educación Infantil: un espacio para crecer como grupo. Revista Iberoamericana de Educación, 71, 133-150.

Sánchez Rodríguez, S. y Santolària Òrrios, A. (2020). Análisis de publicaciones sobre alfabetización inicial desde una perspectiva didáctica. Tejuelo, 32, 229-262. https://doi.org/10.17398/1988-8430.32.229

Santana, R., Alemán, J. A. y López, M. (2017). Leer por placer: ¡lo primero, oír los libros! Aula abierta, 46, 83-90.

Santolària Òrrios, A. (2018). Infants que escriuen sobre protagonistes (sàvies) de contes, infants que hi pensen. Lenguaje y textos, 47, 83-94. https://doi.org/10.4995/lyt.2018.8595

Santolària Òrrios, A. (2019). La secuencia didáctica: un instrumento para escribir textos en educación infantil. Didáctica. Lengua y Literatura, 31, 285-301.

Santolària Òrrios, A. y Ribera, P. (2017). Escrivim. Seqüències didàctiques per a l'escola. Edicions del Bullent.

Santolària Òrrios, A. y Sánchez Rodríguez, S. (2021). Hablar antes de escribir y pensar antes de hablar. Aula de Innovación Educativa, 301, 16-20.

Santos, M. M. (2018). O ensino precoce da escrita: o ditado ao adulto mediado por sequências didáticas com recurso ao quadro interativo [Tesis doctoral. Universidade de Aveiro]. https://ria.ua.pt/handle/10773/26159?locale=pt_PT

Sanz Datzira, A. y Monterde i Farnés, M. (2011). Conversem, llegim i escrivim. Aprenem amb sentit. Articles de Didàctica de la Llengua i la Literatura, 55, 33-41.

Teberosky, A. y Tolchinsky, L. (1995). Más allá de la alfabetización. Santillana.

Tolchinsky, L., Ribera, P. y García-Parejo, I. (2012). Conocimientos iniciales y logros de los alumnos en el aprendizaje de la lectura y la escritura. Cultura y Educación, 24(4), 415-433.

Trigo-Ibáñez, E., Rivera-Jurado, P. y Sánchez-Rodríguez, S. (2020). Using reading alouds in early childhood teacher preparation programs at University of Cádiz. Íkala, 25, 605-624. https://doi.org/10.17533/udea.ikala.v25n03a07

Viera, A. y Barrio, L. (2012). Nacidos para leer... ¿Se nace o se hace? Aula de Infantil, 67, 17-21.

Wells, G. (2015). Dialogic learning: Talking our way into understanding. En T. Dragonas, K. J. Gergen, S. McNamee y E. Tseliou (Eds.). Education as social construction: Contributions to theory, research and practice (pp. 62-91). Taos Institute.

Zamero, M. (2010). La formación en alfabetización inicial de los futuros docentes. Ministerio de Educación de la Nación-Instituto de Formación Docente. http://repositorio.educacion.gov.ar/dspace/handle/123456789/109640 
Zuccalá, G. y Sandbank, A. (2018). Producir textos en los inicios de la alfabetización: el impacto de diferentes enfoques pedagógicos en la expresión evaluativa. Bellaterra Journal of Teaching \& Learning Language \& Literature, 11, 54-73. https://doi.org/10.5565/rev/jtl3.771

\section{ANEXO}

Desarrollo de la secuencia didáctica

\begin{tabular}{|c|c|}
\hline $\begin{array}{l}\text { Desarrollo } \\
\text { de la secuencia }\end{array}$ & Actividades \\
\hline $\begin{array}{l}\text { Fase } 1 \\
\text { Contextualización } \\
\text { Lectura cuento }\end{array}$ & $\begin{array}{l}\text { Sesión } 1 \\
\text { - La docente crea la necesidad de escribir. } \\
\text { - La maestra lee el cuento en voz alta. Establece estrategias de } \\
\text { prelectura y postlectura. Elabora predicciones para verificar la } \\
\text { comprensión del texto. Se analizan el tema y los personajes. Se } \\
\text { discute sobre la moraleja. } \\
\text { - La maestra enmarca la tarea de escritura dentro del proyecto. } \\
\text { - Estrategias: hablar para escribir, lectura en voz alta, comprensión } \\
\text { lectora. } \\
\text { - Tipo de tarea: oral y colectiva. }\end{array}$ \\
\hline \multirow[t]{3}{*}{$\begin{array}{l}\text { Fase } 2 \\
\text { Realización talleres } \\
\text { aprendizaje }\end{array}$} & $\begin{array}{l}\text { Sesión 2-Taller 1: ¿Qué es una ficha técnica? } \\
\text { - Identificación características de la ficha técnica. } \\
\text { - Reconocimiento de los aspectos comunicativos del género: por qué } \\
\text { se escribe, para qué, para quién, dónde, etc. }\end{array}$ \\
\hline & $\begin{array}{l}\text { Sesión 3-Taller 2: ¿Cómo organizamos la información? } \\
\text { - Reconocimiento del contenido y de la estructura. }\end{array}$ \\
\hline & $\begin{array}{l}\text { Sesión 4-Taller 3: ¿Qué palabras usamos? } \\
\text { - Asimilación de las unidades lingüísticas propias del género. } \\
\text { - Estrategias (en los } 3 \text { talleres): hablar para escribir, lectura } \\
\text { compartida, comprensión lectora, dictado al docente. } \\
\text { - Tipo de tarea (en los } 3 \text { talleres): oral y colectiva. }\end{array}$ \\
\hline $\begin{array}{l}\text { Fase } 3 \\
\text { Revisión talleres }\end{array}$ & $\begin{array}{l}\text { Sesión } 5 \\
\text { - Desarrollo de actividades metacognitivas. La maestra pregunta al } \\
\text { grupo por todo lo que hicieron, qué realizaron en cada parte, con } \\
\text { qué materiales, etc. Cuando los niños responden la maestra pega los } \\
\text { flashcards en la pizarra y a continuación se leen. } \\
\text { - Estrategias: hablar para escribir, lectura compartida, comprensión } \\
\text { lectora. } \\
\text { - Tipo de tarea: oral y colectiva. }\end{array}$ \\
\hline $\begin{array}{l}\text { Fase } 4 \\
\text { Escritura texto }\end{array}$ & $\begin{array}{l}\text { Sesión } \mathbf{5} \\
\text { - Los niños escriben el texto mediante el dictado al docente. Dicen a } \\
\text { la maestra lo que debe escribir, esta lo hace en la pizarra. } \\
\text { - Estrategias: hablar para escribir. } \\
\text { - Tipo de tarea: escritura colectiva con dictado al docente. }\end{array}$ \\
\hline
\end{tabular}

